Yu Li, Songbo Guo, Kai Zhao, Catharina Conrad, Caroline Driescher, Vanessa Rothbart, Uwe Schlomann, Helena Guerreiro, Miriam H. Bopp, Alexander König, Barbara Carl, Axel Pagenstecher, Christopher Nimsky and Jörg W. Bartsch*

\title{
ADAM8 affects glioblastoma progression by regulating osteopontin-mediated angiogenesis
}

https://doi.org/10.1515/hsz-2020-0184

Received May 12, 2020; accepted August 18, 2020; published online September 11, 2020

\begin{abstract}
Glioblastoma multiforme (GBM) is the most aggressive type of brain cancer with a median survival of only 15 months. To complement standard treatments including surgery, radiation and chemotherapy, it is
\end{abstract}

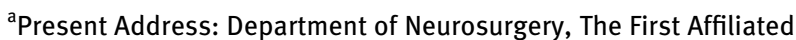
Hospital of Zhengzhou University, 450052 Zhengzhou, China.

\footnotetext{
${ }^{\mathrm{b}}$ Present Address: Diagnostic and Interventional Neuroradiology, University Medical Center Hamburg-Eppendorf, Martinistrasse 52, 20251 Hamburg, Germany

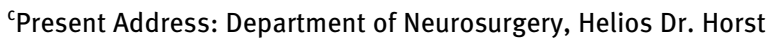
Schmidt Klinik, Ludwig-Erhard-Strasse 100, D-65199 Wiesbaden, Germany
}

*Corresponding author: Jörg W. Bartsch, Department of Neurosurgery, Philipps University Marburg, Baldingerstrasse, D35033 Marburg, Germany; and Center for Mind, Brain and Behavior, Marburg University, Hans-Meerwein-Straße 6, D-35032 Marburg Germany, E-mail: jbartsch@med.uni-marburg.de

Yu Li, Department of Neurosurgery, Philipps University Marburg, Baldingerstrasse, D-35033 Marburg, Germany

Songbo Guo, Department of Neurosurgery, Philipps University Marburg, Baldingerstrasse, D-35033 Marburg, Germany

Kai Zhao, Catharina Conrad, Caroline Driescher, Vanessa Rothbart and Uwe Schlomann, Department of Neurosurgery, Philipps University Marburg, Baldingerstrasse, D-35033 Marburg, Germany Helena Guerreiro, Department of Neurosurgery, Philipps University Marburg, Baldingerstrasse, D-35033 Marburg, Germany

Miriam H. Bopp and Barbara Carl, Department of Neurosurgery, Philipps University Marburg, Baldingerstrasse, D-35033 Marburg, Germany

Alexander König, Department of Diagnostic and Interventional Radiology, Philipps University Marburg, Baldingerstrasse, D-35033 Marburg, Germany

Axel Pagenstecher, Department of Neuropathology, Philipps University Marburg, Baldingerstrasse, D-35033 Marburg, Germany; Center for Mind, Brain and Behavior, Marburg University, Hans-

Meerwein-Straße 6, D-35032 Marburg Germany

Christopher Nimsky, Department of Neurosurgery, Philipps University Marburg, Baldingerstrasse, D-35033 Marburg, Germany; Center for Mind, Brain and Behavior, Marburg University, Hans-Meerwein-Straße 6, D-35032 Marburg Germany essential to understand the contribution of the GBM tumor microenvironment. Brain macrophages and microglia particularly contribute to tumor angiogenesis, a major hallmark of GBM. ADAM8, a metalloprotease-disintegrin strongly expressed in tumor cells and associated immune cells of GBMs, is related to angiogenesis and correlates with poor clinical prognosis. However, the specific contribution of $A D A M 8$ to GBM tumorigenesis remains elusive. Knockdown of $A D A M 8$ in U87 glioma cells led to significantly decreased angiogenesis and tumor volumes of these cells after stereotactic injection into striate body of mice. We found that the angiogenic potential of ADAM8 in GBM cells and in primary macrophages is mediated by the regulation of osteopontin (OPN), an important inducer of tumor angiogenesis. By in vitro cell signaling analyses, we demonstrate that ADAM8 regulates OPN via JAK/STAT3 pathway in U87 cells and in primary macrophages. As $A D A M 8$ is a dispensable protease for physiological homeostasis, we conclude that $A D A M 8$ could be a tractable target to modulate angiogenesis in GBM with minor side-effects.

Keywords: ADAM8; angiogenesis; GBM cell lines; glioblastoma; macrophages; osteopontin; tumor microenvironment.

\section{Introduction}

Glioblastoma multiforme (GBM) is the most common and aggressive type of brain cancer of middle-aged patients with a grim prognosis (Koshy et al. 2012). Until now, the standard clinical treatment of GBM is limited to a combination of surgery, radiation and chemotherapy. Despite continued efforts over several decades to improve patient outcome, GBM remains an incurable disease with a median survival of only 15 months. In 2009, the FDA approved bevacizumab, a drug targeting neovascular formation, for patients with recurrent glioblastomas (Davis 2016). Neo-angiogenesis is critical for tissue growth and repair, and forms the basis for tumor progression (Javan et al. 2019; Viallard and Larrivee 2017). Thus, anti-angiogenic drugs have been widely suggested for clinical cancer treatment. This type of treatment is 
considered to be beneficial as it can not only reduce the blood and nutrient supply of tumor cells in the center of a tumor, but also alleviate edema formation and subsequently neurological symptoms (Berghoff et al. 2014; Takano et al. 2013). However, for bevazizumab, fast mutating tumor cells can rapidly develop resistance by circumventing Vascular Endothelial Growth Factor (VEGF) signaling (Iwamoto et al. 2009), so that alternative routes of blocking angiogenesis are required.

As a large non-tumor cell population, macrophages/ microglia is a prominent cell type of the GBM tumor microenvironment and is a mediator of inflammatory processes. Macrophages can build networks in the tumor that form vascular-like structures to provide oxygen and nutrients for tumor growth, thereby promoting tumor growth (Barnett et al. 2016).

As shown in numerous studies, macrophages can polarize into two extreme types, either M1-like or M2-like. M1-like macrophages are characterized by a proinflammatory and anti-tumor phenotype, while M2-like macrophages are considered as anti-inflammatory and tumor-supporting macrophages (Atri et al. 2018). According to a previous study performed in GBM patients, ADAM8 expression is highly expressed in macrophages/microglia and equally correlated with M1- and M2-like markers, CXCL10 (M1) and CCL13 (M2) (Gjorgjevski et al. 2019).

A disintegrin and metalloproteases (ADAM) constitute a family of multi-domain enzymes with important roles in cell adhesion, migration, proteolysis and signaling. As transmembrane proteins, ADAMs are associated with the process of ectodomain shedding that releases membrane-associated cytokines and receptors into the tumor microenvironment (Kataoka 2009; Murphy 2008). The metalloproteasedisintegrin 8 ( $A D A M 8)$, is a proteolytically active member of the ADAM family (Blobel 2005; Murphy 2008) and was initially identified in macrophages (Yoshida et al. 1990). High expression levels of $A D A M 8$ in tumor cells have been shown to be associated with invasiveness and metastasis of carcinomas, such as breast and pancreatic tumors (Conrad et al. 2017; Romagnoli et al. 2014; Schlomann et al. 2015). For these cancers as well as for glioma, high ADAM8 expression levels correlate with poor patient prognosis (Conrad et al. 2019).

Osteopontin (OPN, gene name SPP1) is a secreted sialoprotein found in all body fluids and expressed in many tissues and cells. OPN is a chemoattractant for many immune cell types including macrophages, dendritic cells, and T cells. Furthermore, OPN enhances B lymphocyte immunoglobulin production and proliferation. When secreted by tumors, for example by glioma cells, OPN has been shown to promote proliferation, migration and angiogenesis via integrin $\alpha \mathrm{v} \beta 3 / \mathrm{PI} 3 \mathrm{~K} / \mathrm{AKT}$ signaling (Ramchandani and Weber 2015; Wang et al. 2011).
In our study, we initially found in GBM tissue that $A D A M 8$ is widely expressed both in neoplastic glioblastoma cells as well as in tumor-associated cells in the tumor microenviroment. We investigated the impact of $A D A M 8$ on GBM progression in vivo by stereotactic injection of $A D A M 8$ deficient glioma cells in the brain of nude mice. Our results suggest a significant contribution of $A D A M 8$ during tumor angiogenesis, as demonstrated in vivo and in vitro by functional analysis of $A D A M 8$ deficient U87 glioma cells and by analyzing primary macrophages isolated from $A D A M 8$ knockout mice. Notably, mice lacking ADAM8 have no evident developmental or pathological defects as confirmed by phenotyping report of ADAM8 knockout mouse (Kelly et al. 2005), which suggests ADAM8 as a potential candidate target protein for GBM therapy with minor expected side effects.

\section{Results}

\section{ADAM8 expression is increased in glioblastoma multiforme and is localized to tumor cells and tumor-associated macrophage/microglia}

A total of 50 GBM tissues obtained from hospitalized patients were examined for $A D A M 8$ expression by qPCR, indicating that $A D A M 8$ expression is significantly higher in GBM compared to normal brain tissue (Figure 1a). Western Blot analyses confirmed higher expression levels of $A D A M 8$ (Figure 1b). Furthermore, $A D A M 8$ localization in GBM tissue was assessed by immunohistochemistry (Figure 1c). In normal brain tissue, $A D A M 8$ expression is hardly detectable and restricted to some neuronal cells. In GBM tissue, $A D A M 8$ expression is significantly higher (approx. 10-fold, $p<0.05$ ) and can be detected in tumor cells, neoplastic endothelial cell with proliferations, and in tumor-associated cells. In order to characterize these cells further, we performed double staining with immune cell markers and found that ADAM8 co-localizes with CD68, a marker for macrophages/ microglia in the brain (Figure 1c, image g). Thus, we conclude that $A D A M 8$ might be functionally important for tumor cells and for tumor-associated macrophages.

To analyze the function of $A D A M 8$ in GBM, we performed loss-of-function experiments in U87 MG cells. Among all human GBM cell lines, U87 MG cells showed the highest level of $A D A M 8$ expression. Briefly, endogenous $A D A M 8$ was knocked down by small hairpin loop RNAs (shRNA) and a number of cell clones $(n=8)$ were obtained with significantly reduced $A D A M 8$ expression levels 
A

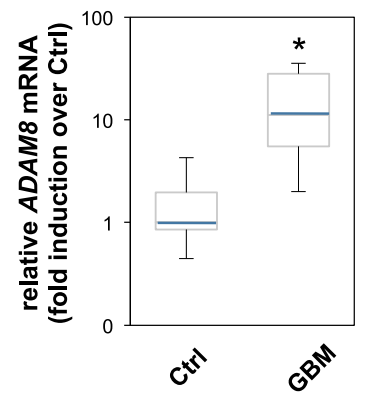

B

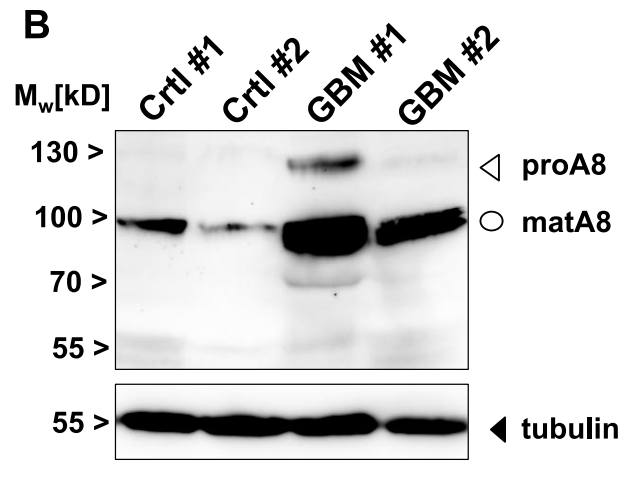

C

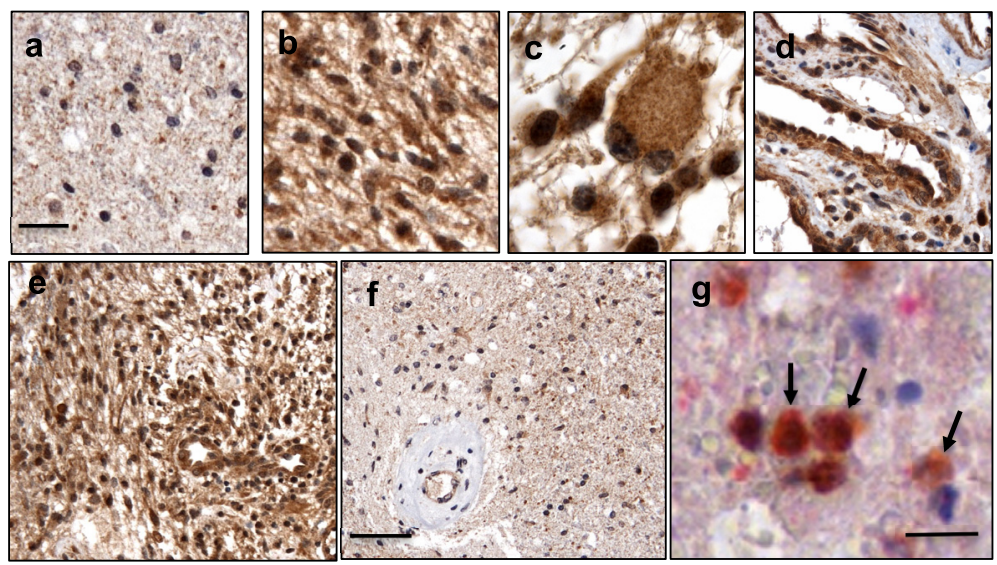

Figure 1: Levels of $A D A M 8$ mRNA expression in GBM tissue.

(A) qPCR analysis of control access CNS tissue ("Ctrl”) and glioblastoma (“GBM") tissue ( $n=5$ for access tissue, $n=50$ for GBM; *, $p<0.05$ ). (B) Western Blot analysis of representative control and GBM tissue samples $(n=2)$. The arrowhead indicates the inactive proform, the circle the active mature ("matA8") form of $A D A M 8$. Note that significantly more mature $A D A M 8$ is detected in GBM tissue. (C) Immunohistochemistry of $A D A M 8$ in normal brain (a) and GBM tissue sections (b-f); $\mathrm{ADAM}^{+}$cells are tumor cells (b-c), endothelial proliferates (d) which can be detected in the core tumor (e) and the infiltration zone (f). (g) Double staining of ADAM 8 (brown) and macrophage marker CD68 (pink) to detect ADAM8 in macrophages/microglia, indicated by arrows. Scale bar in (a), (f): $100 \mu \mathrm{m}$, in (g): $30 \mu \mathrm{m}$. (>50-fold). The cell clone with the highest $A D A M 8$ reduction assessed by qPCR and western blot (Figure 2a) was used for further functional analyses in vitro and in vivo (Figure 2). The proliferation behavior of cells showed no difference (Figure 2b), while cell migration was significantly reduced in U87_shA8 cells (Figure 2c).

\section{$A D A M 8$ reduced angiogenesis and tumor volume in a GBM model}

Using U87 control (scramble, U87_shCtrl) and U87 ADAM8 knockdown (U87_shA8) cell clones, intracranial tumor cell injections into striatum of nude mice were carried out to assess tumorigenesis. To detect tumors, MRI scanning was started three weeks after implantation in weekly intervals and tumor volumes were calculated ( $n=14$ for each group, Figure 2d). All mice injected with U87_shA8 cells survived over an observation time of nine weeks. In contrast, all mice injected with U87_shCtrl cells reached the endpoint criteria or died spontaneously within the observation time (Supplementary Figure 1). Compared to the U87 control group, tumor volumes of U87_A8kd cells were significantly smaller and, in some cases (6 out of 14), tumor cells were completely absent three weeks after implantation (Figure 2a). To assess a possible tumor graft rejection, injection sites were analyzed one week after injection (Figure 2e). Whereas tumors derived from U87_shCtrl cells grew as a compact cell mass after one week, U87_A8kd cells showed a patchy growth characteristic. After staining for vascular structures using CD31 staining, we found significantly less (reduced to $46 \%, p<0.01)$ vascular staining in U87_shA8 $\left(8.7 \times 10^{4} \pm 8\right)$ compared to the U87_shCtrl group (Figure 2e).

\section{$A D A M 8$ regulates $\mathrm{GBM}$ cell angiogenesis via osteopontin}

Given the results of the in vivo experiments, we postulated that U87_shA8 cells could be deficient in angiogenesis induction. To test this hypothesis in vitro, we utilized conditioned medium from U87_shCtrl and U87_shA8 cells for angiogenesis induction of human umbilical vein endothelial cells (HUVEC) and quantified formation of tubes. After $6 \mathrm{~h}$ incubation with the respective cell culture supernatants, tube formation was quantified by counting the number of 

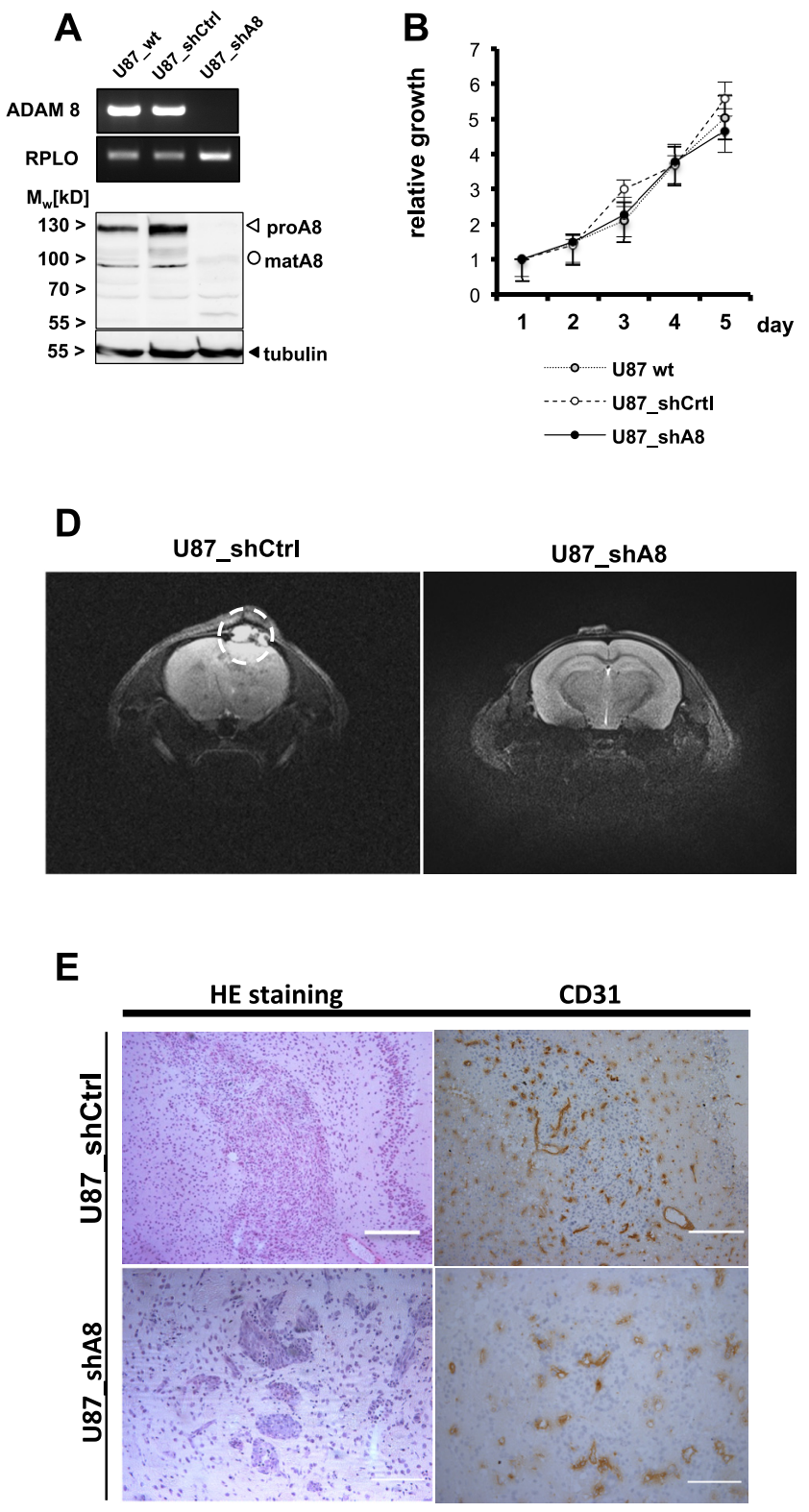

meshes formed (Figure 3a, b). ADAM8 containing supernatants from U87_shCtrl cells induced significantly higher numbers of tubes as compared to supernatants from ADAM8-deficient U87_shA8 cells $(p<0.05)$.

To identify the molecular mechanism by which ADAM8 affects angiogenesis, a differential analysis of cell supernatants from U87_shCtrl and U87_shA8 cells was conducted in an angiogenesis protein profiler array. Array membranes with spotted antibodies against proteins involved in angiogenesis were incubated with conditioned supernatants of U87_shCtrl and U87_shA8 cells and the proteins captured by antibodies on the array membrane were detected by chemiluminescence and quantified

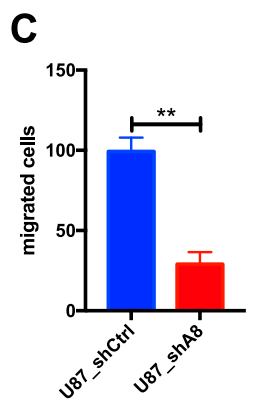

Figure 2: The effect of $A D A M 8$ knockdown in a GBM model.

(A) Knockdown of $A D A M 8$ was confirmed in U87 MG cells by qPCR and Western Blot. In the $A D A M 8$ knockdown clones selected for analysis, no difference in $A D A M 8$

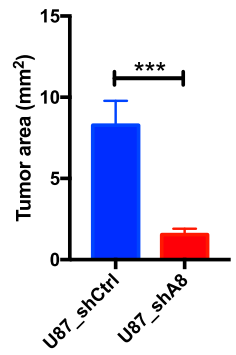

expression compared to U87 wild-type cells was observed. (B) Proliferation of cell clones was comparable in all three U87 cell lines as confirmed by MTT assays. (C) Cell migration was analyzed by scratch assays using U87_shCtrl and U87_shA8 cells. A significant reduction of cell migration was observed in U87 MG cells with ADAM8 knockdown. (D) U87 cells (80.000 in total) were stereotactically injected into the striata of nude mice. 3 weeks after injection (tumor mass circled, injection channel marked by arrow), tumor volumes formed by U87_shA8 cells in the brain is much smaller compared to U87_shCtrl cells $(p<0.01)$.

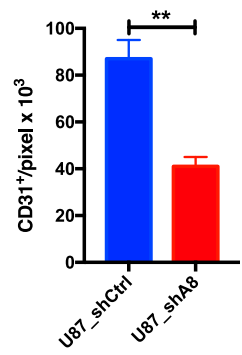
Results are obtained from $n=14$ mice per experimental group. (E) From paraffin sections, vascular structures marked by CD31 staining in the ADAM8 knockdown group were significantly fewer than those of the control group. The scale bar corresponds to $100 \mu \mathrm{m}$. Data from multiple replicates are presented as mean and error bars indicate mean \pm SEM. ${ }^{*} p<0.05$, ${ }^{\star \star} p<0.01,{ }^{\star \star *} p<0.001$, Student's $t$-test.

(Figure 3c). Several candidates were identified, most prominently IL-8 and Osteopontin (OPN, Figure 3c).

Differences in expression levels of OPN between U87_shCtrl and U87_shA8 cells were confirmed by qPCR, suggesting that OPN expression levels are dependent on the ADAM8 gene dosage (Figure 3d).

To provide a direct proof for our hypothesis, we analyzed whether exogenous OPN can be used to recover the deficiency of U87_shA8 cells in angiogenesis assays (Figure 3e, f). Different concentrations of OPN were added to U87 supernatants in angiogenesis assays. When adding IL-8, the reduced angiogenesis capacity of U87_shA8 cells was not restored (data not shown). In contrast, addition of 
A

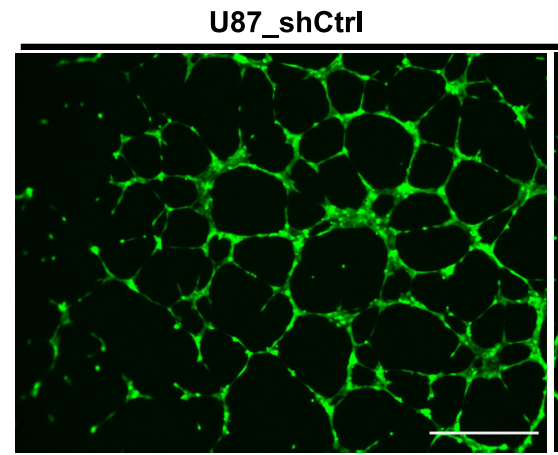

B

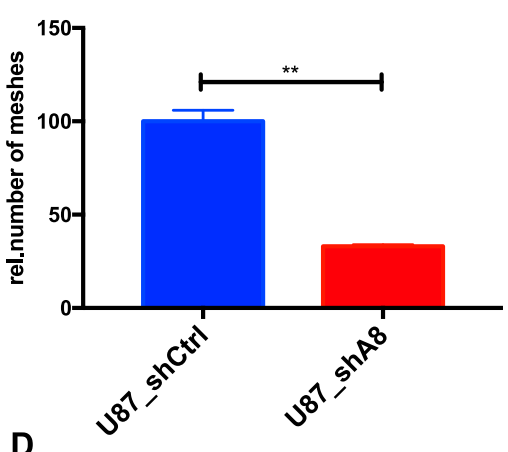

D

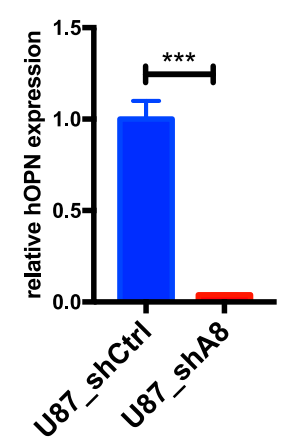

U87_shA8

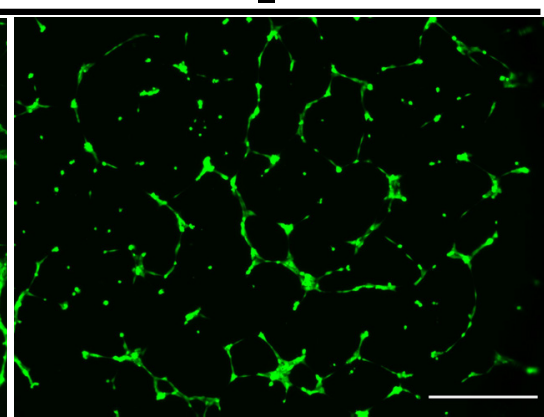

C

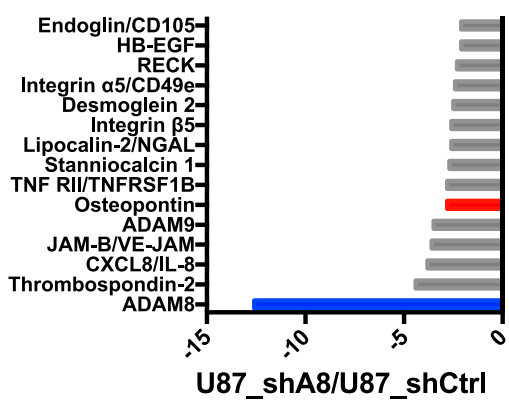

E

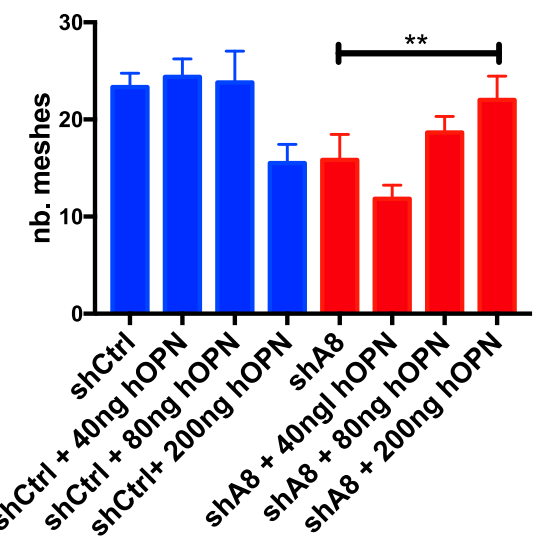

Figure 3: $A D A M 8$ regulates angiogenesis by GBM cells.

(A) HUVEC cells were stimulated with conditioned medium from U87_shCtrl and U87_shA8 cells and tube formation was quantified after $6 \mathrm{~h}$ using Calcein-AM dye. (B) Compared to U87_shCtrl supernatants, HUVEC cell tube formation ability was significantly decreased when U87_shA8 supernatants were applied. (C) Protein Profiler Array to analyze differentially secreted proteins; the expression of osteopontin (OPN), a cytokine related to GBM angiogenesis, was by at least 4-fold decreased. (D) In supernatants of U87_shA8 cells, the mRNA expression of OPN decreased accordingly. (E) Addition of 40, 80 , and $200 \mathrm{ng}$ OPN to angiogenesis assay performed with U87_shCtrl and U87_shA8 supernatants. After addition OPN, the number of meshes formed in the ADAM 8 knockdown group is similar to those ones observed in the ADAM8 wildtype group, but significantly increased compared to ADAM8 knockdown U87 without OPN $(p<0.05)$. The scale bar corresponds to $500 \mu \mathrm{m}$. Data from multiple replicates are presented as mean and error bars indicate mean \pm SD. ${ }^{*} p<0.05$, ${ }^{\star \star} p<0.01,{ }^{\star \star \star} p<0.001$, Student's $t$-test and two-way ANOVA. recombinant OPN at different concentrations caused an increase in the number of meshes formed in the U87_shA group and reached similar values as in the U87_shCtrl group (Figure 3f). As the OPN concentration increases, the number of meshes increases in both groups, although to a smaller extent in U87_shCtrl cells due to saturation effects (Figure 3e).

\section{ADAM8 affects secretion of OPN via the JAK/ STAT3 pathway in GBM cells}

From previous studies it is known that $A D A M 8$ can directly affect intracellular signaling by either shedding of membrane proteins, binding to integrin $\beta 1$, or by direct interaction with intracellular proteins interacting with the ADAM8 cytoplasmic domain. To explore the signaling pathway involved in OPN regulation by $A D A M 8$, we used a panel of pathway inhibitors to target NF-kB, JAK/STAT3, p38, ERK1/2 and AKT/PI3K (Figure 4). U87_shCtrl and U87_shA8 cells were incubated with the respective inhibitors for $24 \mathrm{~h}$ and levels of secreted OPN in cell supernatants were determined by ELISA. While none of the inhibitors affects OPN secretion in U87_shA8 cells, OPN secretion was significantly decreased in U87_shCtrl cells treated with the JAK/STAT3 inhibitor only, whereas other inhibitors such as NF- $\mathrm{BB}$ and p38 inhibitor caused even slightly enhancing effects on OPN secretion (Figure 4). To 
A

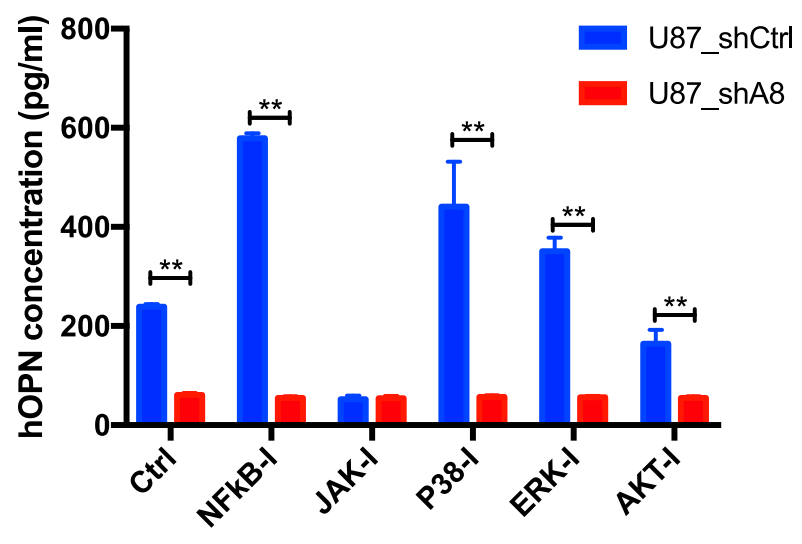

B

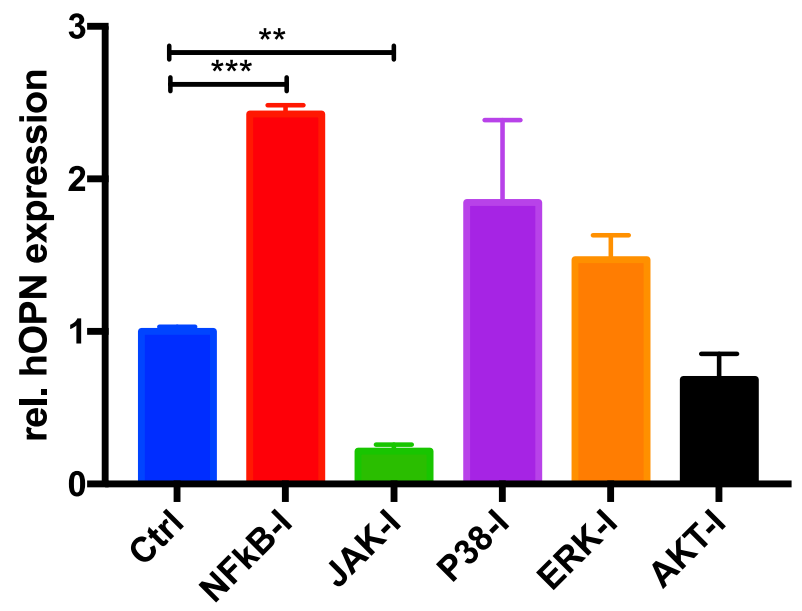

Figure 4: In GBM cells, ADAM8 affects OPN secretion via the JAK/ STAT3 pathway.

(A) Treatment of U87_shCtrl and U87_shA8 cells with inhibitors of NF-KB, JAK/STAT3, p38, ERK1/2, and PI3K/AKT, followed by quantification of OPN release using a specific ELISA. OPN expression of U87_shCtrl and U87_shA8 cells are different even with inhibitor treatment. When inhibiting NF-KB, p38, ERK1/2, OPN expression is not affected or even slightly higher in U87_shCtrl cells compared to non-treated controls. (B) In U87_shCtrl cells treated with JAK/STAT3 inhibitor, OPN expression relative to control conditions (set to 1 ). Data from multiple replicates are presented as mean and error bars indicate mean \pm SD. ${ }^{\star} p<0.05,{ }^{\star \star} p<0.01,{ }^{\star \star \star} p<0.001$, two-way ANOVA and multiple comparison.

clarify the role of the ADAM8 protease function in OPN regulation, U87_shA8 cells were transfected with either active or inactive ( $\mathrm{E}$ to $\mathrm{Q}$ mutation at amino acid position 335) ADAM8. Both, on the mRNA and the protein level, $A D A M 8$ upregulated OPN expression and secretion, however, independent of the protease activity (Supplementary Figure 2).

\section{In macrophages, $A D A M 8$ is involved in the regulation of angiogenesis via OPN}

As shown above (Figure 1c), ADAM8 is additionally expressed in GBM associated macrophages/microglia (GAMMs) [9] which could contribute to tumor angiogenesis in GBM. To analyze if $A D A M 8$ affects OPN expression in these cells similar to tumor cells, we used primary macrophages isolated from wild-type (WT) and ADAM8-deficient mice (Kelly et al. 2005) and no difference in macrophage development was observed (Bartsch et al., unpublished). Primary macrophages were isolated from bone marrow with a purity of $>90 \%$ (Figure $5 \mathrm{a}, \mathrm{b}$ ). Supernatants from macrophages were collected and angiogenesis assays using HUVEC cells were performed. After $6 \mathrm{~h}$ incubation with supernatants from WT and ADAM8-deficient macrophages, HUVEC tube formation was significantly lower in supernatants from ADAM8-deficient macrophages compared to wild-type macrophages (Figure $5 \mathrm{c}-\mathrm{e}$ ). Similar to U87 cells, we analyzed differential proteins secreted from macrophages using a cytokine array. The strongest difference between WT and ADAM8-deficient macrophages was observed for osteopontin (Figure 5f).

\section{ADAM8 regulates OPN via JAK/STAT3 and NF- $K B$ in macrophages}

Expression levels of OPN in WT and A8KO macrophages were confirmed by qPCR (Figure 6a) and ELISA assays (Figure 6b). We further analyzed if OPN added to A8KO macrophages restores angiogenesis; $100 \mathrm{ngl}$ OPN restores the decrease of angiogenic ability caused by ADAM8 deletion but had little effect on WT macrophage supernatant (Figure 6c). Signaling pathway inhibitors were used in macrophages. Protein levels of OPN in cell supernatants were determined by ELISA. Inhibition of JAK/STAT3 and $\mathrm{NF}-\mathrm{\kappa B}$ caused a significant reduction of OPN expression in WT and A8KO macrophages (Figure 6d, e), suggesting that $A D A M 8$ is one of the upstream proteins to regulate OPN expression through JAK/STAT3 and NF-kB.

\section{Discussion}

Expression of $A D A M 8$ has been described to be involved in tumor cell invasion in gliomas (Wildeboer et al. 2006) and 
A BMDM_A8wt

B

BMDM_A8ko
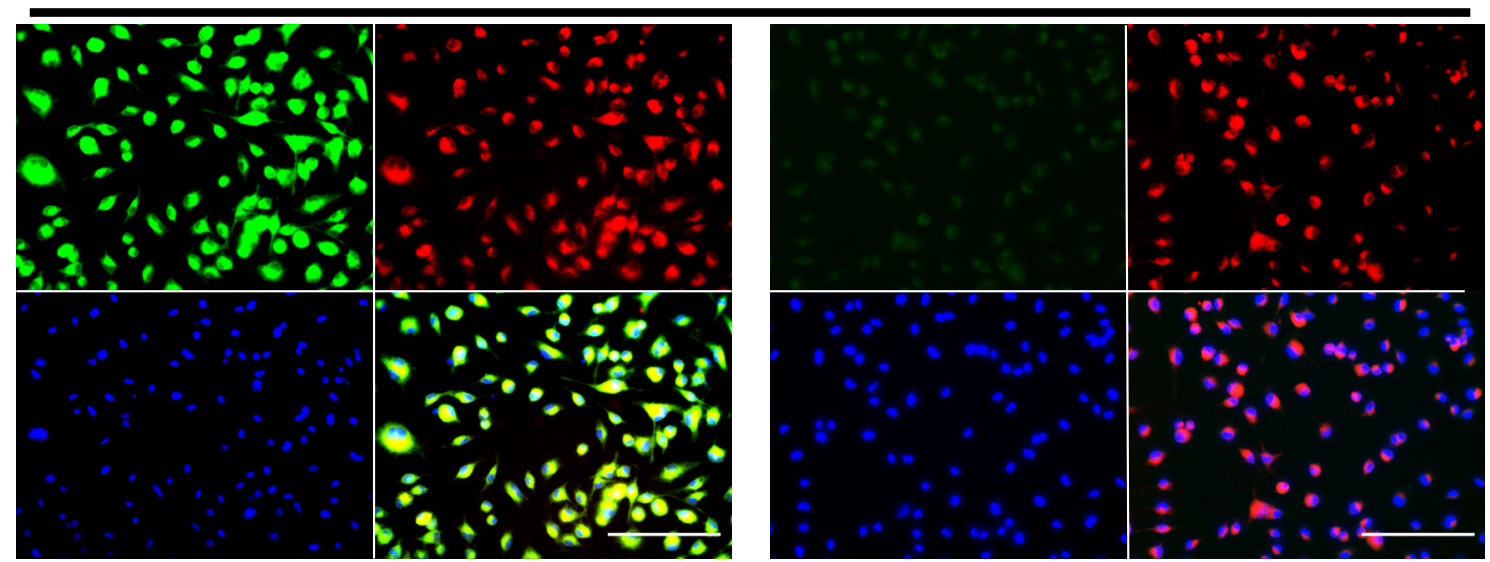

C

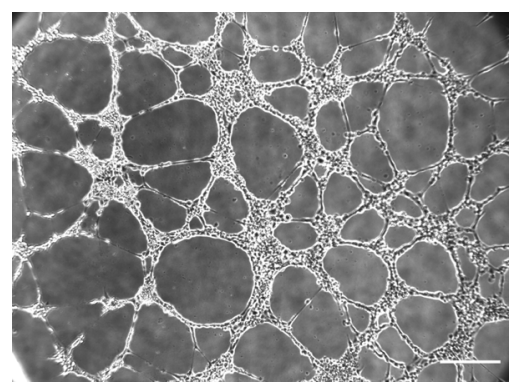

$\mathbf{F}$

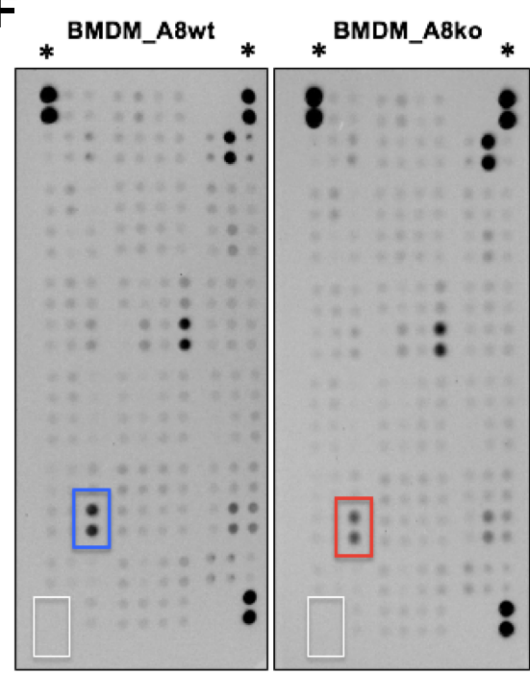

D

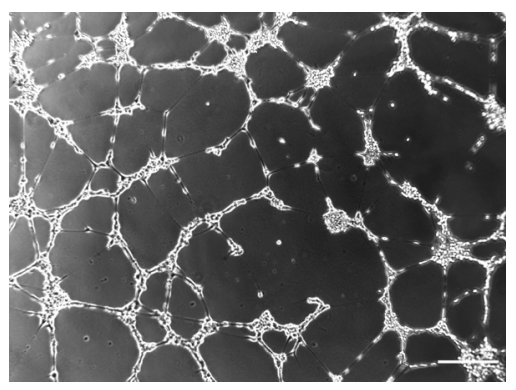

G
E

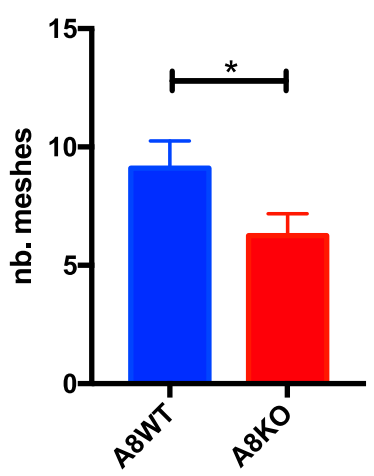

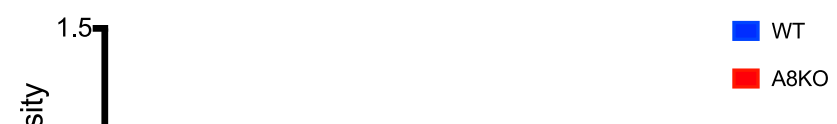

Figure 5: $A D A M 8$ regulates the angiogenic potential of macrophages.

(A, B) Enrichment of bone marrow derived macrophages (BMDM) from WT and ADAM8-deficient (A8KO) mice according to the protocol described in the MandM section. CD11b was used as macrophage marker to quantify purity of macrophage preparation. In WT bone marrow derived macrophages, $A D A M 8$ ( $\mathrm{m}-\mathrm{A} 8$, green) and CD11b (red) stainings are positive. In bone marrow macrophages derived from $A D A M 8$ deficient mice, only CD11b is positive. (C, D) With WT macrophage supernatants, HUVECs form tube-like structure after $6 \mathrm{~h}$, whereas A8KO macrophage supernatants lead to smaller numbers of tubes after 6 h. (E) Number of meshes quantified in 20 viewing fields ( $n=5$ ). Angiogenesis induced from ADAM8-deficient macrophages is significantly decreased. (F, G) Differential analysis of cytokines and angiogenesis factors in supernatants of WT and A8KO macrophages. Note that osteopontin (OPN, blue box for WT, red box for A8ko) shows the biggest difference between the two experimental groups. White boxes, negative controls, asterisks mark the positive controls. Scale bar in (A) and (B) corresponds to $100 \mu \mathrm{m},(C)$ and (D) corresponds to $500 \mu \mathrm{m}$. Data from multiple replicates are presented as mean values and error bars indicate mean \pm SEM. ${ }^{\star} p<0.05,{ }^{\star \star} p<0.01,{ }^{\star \star \star} p<0.001$ by Student's $t$-test. 
A

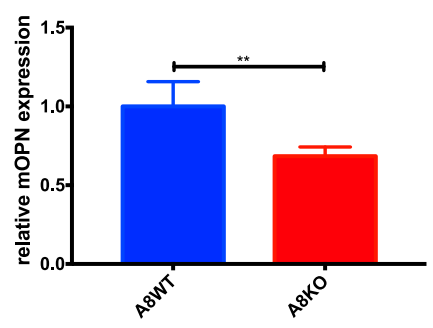

C

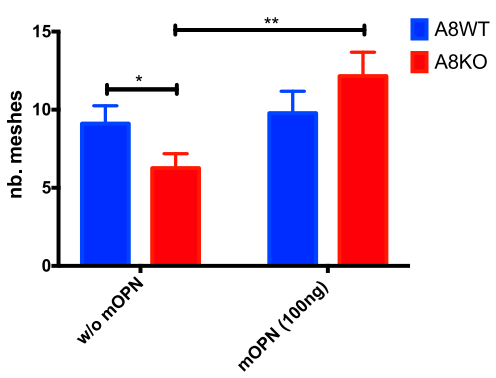

E

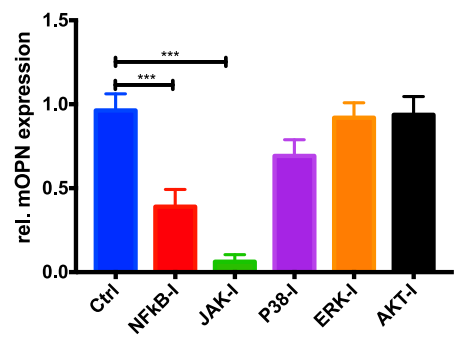

B

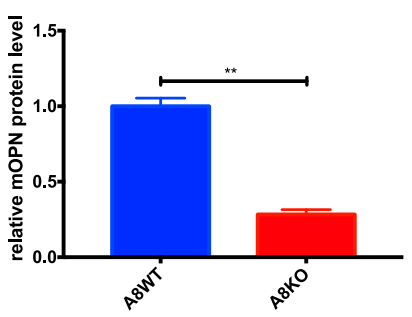

D

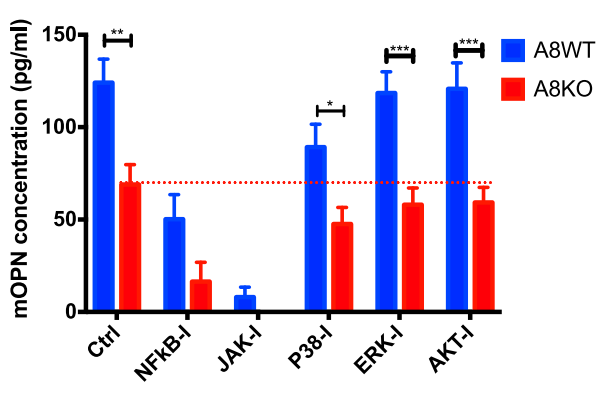

Figure 6: In macrophages, $A D A M 8$ regulates OPN secretion via JAK/STAT3 and NF-KB.

$(A, B)$ Relative OPN expression was determined by qPCR and OPN protein levels in supernatants by ELISA. A8KO macrophages express less OPN than WT macrophages. (C) By adding $100 \mathrm{ng} / \mu \mathrm{LOPN}$, defective angiogenesis of HUVEC cells caused by $A D A M 8$ deficiency can be restored. (D) Treatment of primary macrophages with signaling pathway inhibitors for p38, ERK1/2, PI3K/AKT, NF-KB, JAK/STAT3 followed by quantification of OPN using an ELISA. OPN expression of A8WT and A8KO is decreased after addition NF-KB and JAK/STAT3 inhibitors.

(E) macrophages treated with NF-KB inhibitor and JAK/STAT3 inhibitor. Note that OPN expression is significantly lower than under control conditions (set to 1). Data from multiple replicates are presented as mean and error bars indicate mean \pm SEM. ${ }^{\star} p<0.05,{ }^{\star \star} p<0.01,{ }^{\star \star \star} p<0.001$, Student's $t$-test and two-way ANOVA. high expression levels are unfavorable for glioma patients (He et al. 2012). More recently, ADAM8 expression was correlated to the occurrence of glioma-associated macrophages/microglia (GAMMs, (Gjorgjevski et al. 2019), however the function of $A D A M 8$ in tumor cells and in GAMMs remains elusive and tumor promoting effects in the glioma microenvironment can be postulated, since in all tumor entities described so far, $A D A M 8$ is responsible for tumor invasion, migration, and chemoresistance, leading to poor clinical outcomes (Conrad et al. 2019).

We found that GBM tissues investigated from our tumor collection ( $n=50$ patients) show elevated expression of $A D A M 8$. In GBM tissues, $A D A M 8$, in addition to tumor cell expression, is co-localized with markers for macrophages (CD68), NK cells and neutrophils. By far, the most frequent cell types co-stained with $A D A M 8$ are macrophages, suggesting that $A D A M 8$ is important for tumor cell-macrophage communication in the tumor microenvironment. One of the most important functions of $A D A M 8$ decribed so far, is the contribution to neovascularization and angiogenesis (Guaiquil et al. 2010; Romagnoli et al. 2014). Thus, it is likely that the presence of $A D A M 8$ in GBM is associated with increased angiogenesis. To analyze if angiogenesis induction is based on a systemic effect, we initially investigated tumor cells and macrophages separately in vitro using genetic means to reduce the $A D A M 8$ gene dosage. Our results show that $A D A M 8$ induced in GBM can either be expressed in tumor cells or in GAMMs. As demonstrated previously, there is no association of $A D A M 8$ expression with a certain macrophage polarization state (M1/M2), so we can conclude that potentially both macrophage states could contribute to the observed angiogenesis effect (Dai et al. 2009). As an active metalloprotease, $A D A M 8$ can cleave substrates relevant for angiogenesis, as shown in the context of retinal neovascularization (Guaiquil et al. 2010). However, in this experimental setting, $A D A M 8$ as a sheddase of angiogenesis factors such as Tie-2, VE-cadherin, and CD31 has an 
opposite effect on angiogenesis i.e., blocks angiogenesis by inactivating angiogenesis-related proteins. By investigating the release of angiogenesis related molecules, we noticed that osteopontin is regulated by $A D A M 8$ at the transcriptional level, leading to decreased levels of both, RNA and secreted OPN in tumor cells and macrophages as a result of reduced $A D A M 8$ levels. Thus, $A D A M 8$, via a nonproteolytic function (Supplementary Figure 2), can modulate intracellular signaling in tumor cells and in GAMMs, thereby potentially affecting tumor angiogenesis in GBM patients. We admit that using HUVEC cell angiogenesis provides only a clue to the impact of ADAM8 in GBM angiogenesis, since we analyze a general angiogenic property and not particularly tumor angiogenesis as observed in GBM. However, OPN has been proven to be a potent effector in GBM angiogenesis and our in vivo experiments prove this concept despite these limitations.

Further we confirm that OPN could indeed promote the angiogenesis ability of HUVEC cells, we performed OPN "rescue" assays. When adding OPN to U87 cell supernatant of ADAM 8 knockdown cells, OPN restores the ability of U87 cells to promote angiogenesis. In a study investigating the functional effect of OPN on HUVEC cells (Dai et al. 2009), a concentration of up to $5 \mu \mathrm{M}(44 \mu \mathrm{g} / \mathrm{ml})$ was used to activate PI3K/AKT and ERK1/2 signaling pathways. In our study, we used up to $200 \mathrm{ng}$ of OPN corresponding to $0.2 \mu \mathrm{M}$, a concentration that is highly potent in inducing angiogenesis. For wild-type U87 supernatants, tube formation was only slightly increased compared to wild-type U87 supernatants without OPN. This indicates that at this point the ability of tube formation of HUVECs reached saturation. In contrast, when OPN was added to U87_shA8 cell supernatants, the ability of tube formation reached similar levels than the angiogenesis potential of U87_shCtrl cells. This prompted us to conclude that OPN is an ADAM8-dependent factor essential for HUVEC tube formation and for tumor angiogenesis. In this regard, there are numerous studies supporting the notion that OPN promotes tumor angiogenesis. OPN was found along with VEGF in leukemia, involved in angiogenesis and chemoresistance (Mirzaei et al. 2018). In primitive connective tissue, OPN contributes as a key element to enhance osteogenesis and angiogenesis (Carvalho et al. 2020). In endothelial cells, OPN stimulates angiogenesis via phosphorylation and activation of the PI3K/AKT and ERK1/2 pathway (Ramchandani and Weber 2015). In conclusion, OPN has a general effect on angiogenesis promotion on different types of cells and expression of OPN in GAMMs is particularly essential for angiogenesis in GBM (Guaiquil et al. 2010). In addition, OPN is essential for infiltration of GAMMs in GBM (Wei et al. 2019). In GBM patients, elevated OPN levels were detected in serum samples, and their levels correlate with poor prognosis (Sreekanthreddy et al. 2010)

Our study provides novel evidence that $A D A M 8$ regulates OPN expression and secretion. In order to further understand the mechanism of this regulation, several signal pathway inhibitors were used to block the specific pathways of NF-kB (Berbamine dihydrochloride), JAK/ STAT3 (WP1066), P38 (SB203580), ERK1/2 (U0126), and PI3K/AKT (LY294002). We found that predominantly blocking JAK/STAT3 can significantly reduce expression levels of OPN, while other pathway inhibitors have an opposite (NF-kB) or no effect (p38, ERK1/2, PI3K/AKT) on OPN release in U87_shctrl, but not in U87_shA8 cells. Based on these findings, we can conclude that ADAM8 derived from U87 can promote angiogenesis through OPN via the JAK/STAT3 pathway. STAT3 in particular was described as an important factor for glioma infiltration and growth (Priester et al. 2013) and the activation of this pathway by $A D A M 8$ presents a novel finding.

Interestingly, a similar regulation of OPN by ADAM 8 was also found in bone-marrow derived macrophages. To analyze this, we used primary macrophages from ADAM8deficient mice as the most suitable cell model. In these macrophages, not selected for a certain polarization type, expression of OPN decreased in the absence of ADAM8, so that the ability to stimulate angiogenesis in vitro decreased accordingly. Analogous to the experiments with U87 cells, exogenous addition of OPN restores the observed decrease of angiogenesis caused by ADAM8 deficiency, demonstrating that an $A D A M 8$ to OPN signaling cascade is mode of action in these cell types with regard to angiogenesis. Considering macrophages as an eminent cell type in the microenvironment of GBM, elimination of ADAM8 in the tumor microenvironment could cause deprivation of OPN, thereby reducing neovascularization of the tumor blood supply. In contrast to U87 cells, OPN secretion in WT and in ADAM8-deficient macrophages can be reduced by the JAK/ STAT3 inhibitor. This suggests that there is an ADAM8dependent and -independent signaling for JAK/STAT3 activation in macrophages. However, the ADAM8-independent OPN regulation occurs almost at a very basal level of expression. In macrophages, there is an additional effect of NF- $\mathrm{KB}$ inhibition on OPN expression.

So far it was unknown how ADAM8 affects OPN expression and secretion. We have seen that the effect of $A D A M 8$ on OPN is independent of its metalloprotease function. Rather, the disintegrin domain and signaling via the cytoplasmic domain seem to be involved in OPN regulation. A few clues came from previous research. In our previous studies of pancreatic cancer, we found that the expression of $A D A M 8$ in pancreatic cancer cell lines Panc1 
and AsPC-1 is related to migration and apoptosis. Invasiveness of pancreatic ductal carcinoma induced by activation of PI3K/AKT and ERK1/2 suggested that $A D A M 8$ is highly correlated with PI3K/AKT and ERK1/2 signaling pathways (Schlomann et al. 2015). A previous study found that $A D A M 8$ promotes migration and invasion of chondrosarcomas by activating NF-kB/MMP-13 (Liu et al. 2019). JAK/STAT3 is related to angiogenesis in some studies (Barry et al. 2007; Valdembri et al. 2002; Xue et al. 2017). By ELISA analysis, we found that the amount of secreted OPN in macrophages stimulated by JAK/STAT3 and NF- $\mathrm{kB}$ inhibitors decreased significantly, suggesting that OPN is regulated by these pathways. As the major target protein of $A D A M 8$, we identified OPN regulated by ADAM8 via JAK/ STAT3 signaling in GBM cells and in macrophages, thereby revealing a potential role of $A D A M 8$ in angiogenesis.

In summary, our study identified $A D A M 8$ as a relevant metalloprotease-disintegrin in GBM. Since $A D A M 8$ is present in tumor cells and in GAMMs, we hypothesize that $A D A M 8$ is an important modulator of the tumor microenvironment. In this respect, the extent of angiogenesis we have analyzed in vivo could be underestimated since we expect GAMM-derived OPN to additionally contribute to tumor angiogenesis in GBM. Considering that OPN derived from tumor cells is an important factor for recruitment of macrophages from the periphery (Wei et al. 2019), the additional OPN levels secreted from macrophages could form an angiogenesis-supporting microenvironment in GBM. Further studies will prove for these mechanisms in an animal model and further exploit these mechanisms in vivo with the goal to establish $A D A M 8$ as a drug target in glioblastoma.

\section{Materials and methods}

\section{Patient specimens}

Approval from the local ethics committee (file number 185/11) was obtained to collect tumor tissue samples from patients who underwent surgical resection of GBM after providing written consent. Tumor tissue was either shock-frozen in liquid nitrogen (biochemical analyses) or embedded in paraffine for immunohistochemistry.

\section{Cells}

U87 MG cells were obtained from ATCC and their authenticity was checked by STR profiling and karyotype analysis with X- and Y-specific FISH probes. Cells were grown in DMEM with 10\% FCS. HUVEC cells were purchased from Promo Cell (Heidelberg, Germany) and maintained according to the manufacturer's instructions.
To generate U87 MG cells with an ADAM8 knockdown, cells were transfected with four shADAM8 constructs (TF314948, Origene, Rockville, MD, USA with the target sequence 5'- GCGGCACCTGCATGACAACGTACAGCTCA-3') cloned into pRFP-C-RS vector using Lipofectamine LTX (Invitrogen, UK). Twenty-four hours after transfection, cells were treated with the respective selection antibiotic ( $1 \mathrm{mg} / \mathrm{ml}$ puromycin). Around 15 cell clones were selected and successful knockdown of ADAM8 was confirmed. For generation of control cell clones, a construct with a scramble shRNA (target sequence 5'-GCACTACCAGAGCTAACT CAGATAGTACT-3') sequence was used. ADAM8 expression in single U87 MG cell clones was analyzed by qRT-PCR, western blotting and ELISA.

\section{Mice}

ADAM8-deficient mice are kept on a C57B1/6 background for at least 10 generations. For genotyping, diagnostic PCR assays were performed as described (Schlomann et al. 2015). For in vivo experiments, sixweek-old athymic mice were obtained from Harlan (Indianapolis, USA). All animal experiments were performed in accordance with the German laws on the protection of animals ("Tierschutzgesetz") and with permission by the local authorities (Regierungspräsidium Gießen V54-19c2015(1) MR20/18, and G60_2016).

\section{Mouse bone marrow-derived macrophage isolation and culture}

Mouse macrophages were isolated from mouse bone marrow. Mice were sacrificed by cervical dislocation, and femur and tibia were removed to flush bone marrow out with completed culture medium (10\% FCS, $20 \mathrm{ng} / \mathrm{ml}$ MCSF, DMEM) using a $21 \mathrm{G}$ needle. After separating the cells using a cell strainer (pore size $100 \mu \mathrm{m}$ ), cells were seeded in a petri dish in $10 \mathrm{ml}$ complete medium. At day $4,5 \mathrm{ml}$ of the complete medium were replaced. After 7 days of incubation, macrophages were used for functional experiments.

\section{Proliferation assays}

Cells were plated at 5000 cells/well in 96-well plates and cultured for 1, 2, 3, 4 and 5 days. On the indicated days, 3-(4,5-Dimethylthiazol2-yl)-2,5-diphenyltetrazolium bromide (MTT) reagent (AMRESCO, Solon, OH, USA) was added and incubated for $4 \mathrm{~h}$ at $37{ }^{\circ} \mathrm{C}, 5 \% \mathrm{CO}_{2}$. The supernatant was discarded and replaced with $200 \mu \mathrm{l}$ DMSO (SigmaAldrich Co., St. Louis, MO, USA) to dissolve the formazan product. Absorbance at $570 \mathrm{~nm}$ was determined in a 96-well-plate reader (BMG Labtech, Offenburg, Germany).

\section{Scratch assays}

Cell migration was determined by scratch assays in vitro in cultureinsert microdishes (Ibidi GmbH, Planegg, Germany) according to the manufacturer's instructions. Cells were seeded into each chamber at densities of $1 \times 10^{5}$ cells $/ \mathrm{ml}$ in $100 \mu$ l DMEM medium with $10 \%$ FCS. After incubation at $37{ }^{\circ} \mathrm{C}, 5 \% \mathrm{CO}_{2}$ for $24 \mathrm{~h}$ and serum starving for another $6 \mathrm{~h}$, inserts were removed and $1 \mathrm{ml}$ DMEM medium with $2 \%$ FCS was added into each dish. Images were taken immediately after insert removal and $18 \mathrm{~h}$ later. Rates of cell migration were determined 
by counting cells in the gaps ( $n=3$ viewing fields per plate in three independent experiments) using Image J software.

\section{Immunofluorescence microscopy}

1000 cells were seeded per well in an eight well chamber on glass (LabTek,4808), fixed and stained with antibodies (CD11b: Thermo Fisher, MA1-80091; mouse ADAM8: Biorbyt, orb4376; human ADAM8: R\&D Systems, AF1031).

\section{Angiogenesis tube formation assay}

$1 \times 10^{6}$ macrophages/U87 cells (ADAM8 deficient compared to respective control) were seeded in a six well in $1 \mathrm{ml}$ of a 1:1 mix serum free DMEM and ECM MEDIUM (Promo Cell, C-22010). After 24 h, supernatant was collected and cleared by centrifugation at $1000 \mathrm{rpm}$ for 5 min. $4 \times 10^{5}$ HUVECs per well were seeded on Angiogenesis Plate (IBIDI, 81506). After particular time points (Macrophages: $6 \mathrm{~h}$; U87: $15 \mathrm{~h}$ ), three pictures (center, upper left and lower right) were taken from each well and meshes formed were counted. For the OPN "rescue" assay, $2.5 \mathrm{ng} / \mathrm{ml}$ OPN (PeproTech, 120-35) was added to macrophage and $50 \mathrm{ng} / \mathrm{ml}$ OPN to U87 supernatants.

\section{Cytokine array}

A membrane-based antibody array [Proteome Profiler ${ }^{\circledR}$ Mouse XL Cytokine Array ARY028, RandD Systems, Abingdon, UK] was used to identify cytokine expression levels under different conditions, according to the manufacturer's protocol. Briefly, conditioned supernatants of U87_shA8 and U87_shCtrl cells were incubated with a biotinylated antibody mix for detection on the nitrocellulose membrane by chemiluminescence. Quantification of dot optical intensities was performed using Image J software.

\section{Real-time quantitative PCR}

To determine gene expression, real-time quantitative PCR with SYBR Green (Precision ${ }^{\circledR}$ FAST master mix) on StepOnePlus RealTime PCR System was performed using commercially available primers for m-ADAM8 (Qiagen, Hilden, Germany), m-SPP-1 (Qiagen) h-ADAM8 (Qiagen), h-SPP-1 (Apara Bioscience, Freiburg, Germany), RPLPO (Qiagen) was used as reference gene for normalization. Relative gene expression was calculated using comparative $2^{-\Delta \Delta \mathrm{Ct}}$ method.

\section{ELISA}

Human and mouse osteopontin levels in cell culture supernatants were determined by ELISA kits for human OPN (R\&D systems, DY1433, Southampton, UK) and mouse OPN (R\&D Systems, DY441) according to the manufacturer's instructions.

\section{Statistical analysis}

Unpaired two-tailed Student's $t$-test was used to compare values from two independent groups. For multiple comparisons, Two-way ANOVA and multiple comparisons were used. Data from multiple replicates are presented as mean and error bars indicate mean \pm SEM from at least three independent samples. Based on the obtained results, the data were considered not significant $(p>0.05)$, significant $\left({ }^{\star} p<0.05\right)$, highly significant $\left({ }^{\star \star} p<0.01\right)$ or very highly significant $\left({ }^{\star \star} p<0.001\right)$. Calculations were performed using the GraphPrism statistical analysis software.

Acknowledgments: We thank the $7 \mathrm{~T}$ small animal MRI core facility at Marburg University for their help with imaging and Agnes Schäfer and Lara Evers for help with ADAM8 expression analyses. Work was funded by the von-BehringRöntgen Stiftung (58-0044 to JWB and 64-0018 to MHB) and by the DFG (BA 1606/3-1 to JWB).

Author contribution: All the authors have accepted responsibility for the entire content of this submitted manuscript and approved submission.

Research funding: We thank the 7T small animal MRI core facility at Marburg University for their help with imaging. Work was funded by the von-Behring-Röntgen Stiftung (580044 to JWB and 64-0018 to MHB) and by the DFG (BA 1606/3-1 to JWB)

Conflict of interest statement: The authors declare no conflicts of interest regarding this article.

\section{References}

Atri, C., Guerfali, F.Z., and Laouini, D. (2018). Role of human macrophage polarization in inflammation during infectious diseases. Int. J. Mol. Sci. 19: 6.

Barnett, F.H., Rosenfeld, M., Wood, M., Kiosses, W.B., Usui, Y., Marchetti, V., Aguilar, E., and Friedlander, M. (2016). Macrophages form functional vascular mimicry channels in vivo. Sci. Rep. 6: 36659.

Barry, S.P., Townsend, P.A., Latchman, D.S., and Stephanou, A. (2007). Role of the JAK-STAT pathway in myocardial injury. Trends Mol. Med. 13: 82-89.

Berghoff, A.S., Sax, C., Klein, M., Furtner, J., Dieckmann, K., Gatterbauer, B., Widhalm, G., Rudas, M., Zielinski, C.C., Bartsch, R., et al. (2014). Alleviation of brain edema and restoration of functional independence by bevacizumab in brain-metastatic breast cancer: a case report. Breast Care 9: 134-136.

Blobel, C.P. (2005). ADAMs: key components in EGFR signalling and development. Nat. Rev. Mol. Cell Biol. 6: 32-43.

Carvalho, M.S., Silva, J.C., Hoff, C.M., Cabral, J.M.S., Linhardt, R.J., da Silva, C.L., and Vashishth, D. (2020). Loss and rescue of osteocalcin and osteopontin modulate osteogenic and 
angiogenic features of mesenchymal stem/stromal cells. J. Cell. Physiol. 235: 7496-7515.

Conrad, C., Benzel, J., Dorzweiler, K., Cook, L., Schlomann, U., Zarbock, A., Slater, E.P., Nimsky, C., and Bartsch, J.W. (2019). $A D A M 8$ in invasive cancers: links to tumor progression, metastasis, and chemoresistance. Clin. Sci. 133: 83-99.

Conrad, C., Götte, M., Schlomann, U., Roessler, M., Pagenstecher, A., Anderson, P., Preston, J., Pruessmeyer, J., Ludwig, A., Li, R., et al. (2018). ADAM8 expression in breast cancer derived brain metastases: functional implications on MMP-9 expression and transendothelial migration in breast cancer cells. Int. J. Canc. 142: 779-791.

Dai, J., Peng, L., Fan, K., Wang, H., Wei, R., Ji, G., Cai, J., Lu, B., Li, B., Zhang, D., et al. (2009). Osteopontin induces angiogenesis through activation of PI3K/AKT and ERK1/2 in endothelial cells. Oncogene 28: 3412-3422.

Davis, M.E. (2016). Glioblastoma: overview of disease and treatment. Clin. J. Oncol. Nurs. 20: S2-S8.

Gjorgjevski, M., Hannen, R., Carl, B., Li, Y., Landmann, E., Buchholz, M., Bartsch, J.W., and Nimsky, C. (2019). Molecular profiling of the tumor microenvironment in glioblastoma patients: correlation of microglia/macrophage polarization state with metalloprotease expression profiles and survival. Biosci. Rep. 39: 6.

Guaiquil, V.H., Swendeman, S., Zhou, W., Guaiquil, P., Weskamp, G., Bartsch, J.W., and Blobel, C.P. (2010). ADAM8 is a negative regulator of retinal neovascularization and of the growth of heterotopically injected tumor cells in mice. J. Mol. Med. 88: 497-505.

He, S., Ding, L., Cao, Y., Li, G., Deng, J., Tu, Y., and Wang, B. (2012). Overexpression of a disintegrin and metalloprotease 8 in human gliomas is implicated in tumor progression and prognosis. Med. Oncol. 29: 2032-2037.

Iwamoto, F.M., Abrey, L.E., Beal, K., Gutin, P.H., Rosenblum, M.K., Reuter, V.E., DeAngelis, L.M., and Lassman, A.B. (2009). Patterns of relapse and prognosis after bevacizumab failure in recurrent glioblastoma. Neurology 73: 1200-1206.

Javan, M.R., Khosrojerdi, A., and Moazzeni, S.M. (2019). New insights into implementation of mesenchymal stem cells in cancer therapy: prospects for anti-angiogenesis treatment. Frontiers in Oncology 9: 840.

Kataoka, H. (2009). EGFR ligands and their signaling scissors, ADAMs, as new molecular targets for anticancer treatments. J. Dermatol. Sci. 56: 148-153.

Kelly, K., Hutchinson, G., Nebenius-Oosthuizen, D., Smith, A.J., Bartsch, J.W., Horiuchi, K., Rittger, A., Manova, K., Docherty, A.J., and Blobel, C.P. (2005). Metalloprotease-disintegrin ADAM8: expression analysis and targeted deletion in mice. Dev. Dynam. 232: 221-231.

Koshy, M., Villano, J.L., Dolecek, T.A., Howard, A., Mahmood, U., Chmura, S.J., Weichselbaum, R.R., and McCarthy, B.J. (2012). Improved survival time trends for glioblastoma using the SEER 17 population-based registries. J. Neuro Oncol. 107: 207-212.

Liu, Y., Li, Z.H., Zhang, L., and Lu, S.B. (2019). ADAM8 promotes chondrosarcoma cell migration and invasion by activating the NF-kappaB/MMP-13 signaling axis. Anti Canc. Drugs 30: e0790.

Mirzaei, A., Mohammadi, S., Ghaffari, S.H., Yaghmaie, M., Vaezi, M., Alimoghaddam, K., and Ghavamzadeh, A. (2018). Osteopontin b and c splice isoforms in leukemias and solid tumors: angiogenesis alongside chemoresistance. Asian Pac. J. Cancer Prev. 19: 615-623.

Murphy, G. (2008). The ADAMs: signalling scissors in the tumour microenvironment. Nat. Rev. Canc. 8: 929-941.
Priester, M., Copanaki, E., Vafaizadeh, V., Hensel, S., Bernreuther, C., Glatzel, M., Seifert, V., Groner, B., Kogel, D., and Weissenberger, J. (2013). STAT3 silencing inhibits glioma single cell infiltration and tumor growth. Neuro Oncol. 15: 840-852.

Ramchandani, D. and Weber, G.F. (2015). Interactions between osteopontin and vascular endothelial growth factor: implications for cancer. Biochim. Biophys. Acta 1855: 202-222.

Romagnoli, M., Mineva, N.D., Polmear, M., Conrad, C., Srinivasan, S., Loussouarn, D., Barillé-Nion, S., Georgakoudi, I., Dagg, Á294.

Schlomann, U., Koller, G., Conrad, C., Ferdous, T., Golfi, P., Garcia, A.M., Hofling, S., Parsons, M., Costa, P., Soper, R., et al. (2015). ADAM8 as a drug target in pancreatic cancer. Nat Commun 6: 6175.

Sreekanthreddy, P., Srinivasan, H., Kumar, D.M., Nijaguna, M.B., Sridevi, S., Vrinda, M., Arivazhagan, A., Balasubramaniam, A., Hegde, A.S., Chandramouli, B.A., et al. (2010). Identification of potential serum biomarkers of glioblastoma: serum osteopontin levels correlate with poor prognosis. Canc. Epidemiol. Biomarkers Prev. 19: 1409-1422.

Takano, S., Kimu, H., Tsuda, K., Osuka, S., Nakai, K., Yamamoto, T., Ishikawa, E., Akutsu, H., Matsuda, M., and Matsumura, A. (2013). Decrease in the apparent diffusion coefficient in peritumoral edema for the assessment of recurrent glioblastoma treated by bevacizumab. Acta Neurochir.: 185-189, https://doi.org/10. 1007/978-3-7091-1434-6_34.

Valdembri, D., Serini, G., Vacca, A., Ribatti, D., and Bussolino, F. (2002). In vivo activation of JAK2/STAT-3 pathway during angiogenesis induced by GM-CSF. Fed. Am. Soc. Exp. Biol. J. 16: 225-227.

Viallard, C. and Larrivee, B. (2017). Tumor angiogenesis and vascular normalization: alternative therapeutic targets. Angiogenesis 20 : 409-426.

Wang, Y., Yan, W., Lu, X., Qian, C., Zhang, J., Li, P., Shi, L., Zhao, P., Fu, Z., Pu, P., et al. (2011). Overexpression of osteopontin induces angiogenesis of endothelial progenitor cells via the avbeta3/ $\mathrm{PI} 3 \mathrm{~K} / \mathrm{AKT} / \mathrm{eNOS} / \mathrm{NO}$ signaling pathway in glioma cells. Eur. J. Cell Biol. 90: 642-648.

Wei, J., Marisetty, A., Schrand, B., Gabrusiewicz, K., Hashimoto, Y., Ott, M., Grami, Z., Kong, L.Y., Ling, X., Caruso, H., et al. (2019). Osteopontin mediates glioblastoma-associated macrophage infiltration and is a potential therapeutic target. J. Clin. Invest. 129: 137-149.

Wildeboer, D., Naus, S., Amy Sang, Q.X., Bartsch, J.W., and Pagenstecher, A. (2006). Metalloproteinase disintegrins ADAM8 and $A D A M 19$ are highly regulated in human primary brain tumors and their expression levels and activities are associated with invasiveness. J. Neuropathol. Exp. Neurol. 65: 516-527.

Xue, C., Xie, J., Zhao, D., Lin, S., Zhou, T., Shi, S., Shao, X., Lin, Y., Zhu, B., and Cai, X. (2017). The JAK/STAT3 signalling pathway regulated angiogenesis in an endothelial cell/adipose-derived stromal cell co-culture, 3D gel model. Cell Prolif. 50, https://doi. org/10.1111/cpr.12307.

Yoshida, S., Setoguchi, M., Higuchi, Y., Akizuki, S., and Yamamoto, S. (1990). Molecular cloning of cDNA encoding MS2 antigen, a novel cell surface antigen strongly expressed in murine monocytic lineage. Int. Immunol. 2: 585-591.

\section{Supplementary Material}

The online version of this article offers supplementary material (https://doi.org/10.1515/hsz-2020-0184). 